\title{
Early stages of age-related macular degeneration: an immunofluorescence and electron microscopy study
}

\author{
Theo L van der Schaft, Cornelia M Mooy, Wim C de Bruijn, Paul T V M de Jong
}

\begin{abstract}
In subretinal neovascularisation capillaries originating from the choriocapillaris must cross Bruch's membrane to reach the subretinal pigment epithelial space. Thus gaps in Bruch's membrane have to be formed before subretinal neovascularisation. Histological examination of eyes with subretinal neovascularisation or disciform scars has shown macrophages adjacent to thin areas and ruptures in Bruch's membrane. This has been interpreted as phagocytosis of Bruch's membrane. The purpose of this study was to investigate whether immune complex depositions can be detected in maculae with early stages of agerelated macular degeneration and to explain the macrophage reaction before the disciform reaction. A series of 20 human maculae were examined by direct immunofluorescence light microscopy to detect the presence of immune complexes with antibodies directed against immunoglobulins, fibrinogen, and complement factors. Transmission electron microscopy on several maculae was performed to identify the macrophages. Macrophages were observed in close relation to the readily recognisable long spacing collagen, which suggested that long spacing collagen was selectively internalised by these cells. Definite immune complex depositions were not found in basal laminar deposits or drusen. Linear deposits of fibrinogen and complement were frequently found in the outer collagenous zone of Bruch's membrane. However, because of the absence of immunoglobulins, it seems unlikely that these non-specific deposits might cause chemoattraction of macrophages and play a role in the initial phase of the development of subretinal neovascularisation and disciform macular degeneration.
\end{abstract}

(Brf Ophthalmol 1993; 77: 657-661)

Erasmus University

Rotterdam, The

Netherlands

Institute of

Ophthalmology

TL van der Schaft

C M Mooy

P T V M de Jong

Institute of Pathology C M Mooy

W C de Bruijn

Correspondence to:

Dr Paul de Jong, Institute of

Ophthalmology, Erasmus

University Rotterdam, PO

Box 1738, 3000 DR

Rotterdam, The Netherlands.

Accepted for publication

29 April 1993 considered most likely.'

The presence of a basal laminar deposit (BLD), which is a subretinal pigment epitheliai deposit of extracellular materiàl,,$^{2-4}$ or numerous large hard drusen can eventually lead to the development of atrophy of both the retinal pigment epithelium (RPE) and photoreceptor cells, so-called geographic atrophy. ${ }^{5}$ Soft drusen and BLD are often associated with subretinal neovascularisation, ${ }^{46}$ which eventually results in the development of the exudative end stage of ARMD and leads functionally to deterioration of central vision.

Light microscopically, BLD has been found in aged maculae and is often referred to as abundant basement membrane material, ${ }^{2-4}$ based upon its localisation between the RPE cell membrane and its basement membrane. A thick layer of BLD is usually seen in maculae with RPE degeneration, geographic atrophy, or disciform macular degeneration. ${ }^{347}$

In subretinal neovascularisation, capillaries originating from the choriocapillaris must cross Bruch's membrane, which normally forms a firm and continuous mechanical barrier in the macula, to reach the sub-RPE space. ${ }^{5}$ Therefore a passage through Bruch's membrane has to be formed before neovascularisation. Capillaries that pass Bruch's membrane at the disc usually do not lead to ARMD. Histological examination of eyes with subretinal neovascularisation or disciform scars has shown macrophages adjacent to the thin areas and ruptures in Bruch's membrane. ${ }^{8}$ This has been interpreted as the breakdown of Bruch's membrane by these macrophages. ${ }^{8-10}$

In general, macrophages are attracted by foreign bodies or chemical substances such as immune complexes. ${ }^{112}$ The phagocytic action of macrophages in eyes with ARMD results in the digestion of the outer collagenous zone (OCZ) of Bruch's membrane ${ }^{13}$ preferentially when BLD or drusen are already present. ${ }^{10}$ However, it is not known why macrophages are attracted to, and apparently digest, Bruch's membrane in patients with early stages of ARMD.

The purpose of this study was to investigate whether immune complex deposits can be detected in maculae with early stages of ARMD and to explain the assumed macrophage reaction before the disciform reaction. We examined a series of human maculae by direct immunofluorescence light microscopy with antibodies against immunoglobulins, fibrinogen, and complement factors. Transmission electron microscopy was performed to identify the macrophages.
Materials and methods

The maculae of 20 human eyes from 13 subjects were obtained at autopsy or after surgical enucleation for intraocular melanoma. The ages 
of the subjects ranged from 41 to 96 years (mean 74, SD19.6)

The maculae were dissected from the globes and divided into two equal halves in the direction of the optic disc. One half was snap frozen in isopentane $\left(-70^{\circ} \mathrm{C}\right)$ and stored in liquid nitrogen for immunofluorescence studies. Three series of frozen sections $(5 \mu \mathrm{m})$ taken at $50 \mu \mathrm{m}$ intervals were prepared and mounted on cleaned glass slides. After fixation with acetone the sections were air dried. The slides were placed in a Sequenza immunostaining workstation (Shandon Scientific Ltd, Astmoor Rancorn, Cheshire, UK). Primary antibodies included fluorescein isothiocyanate conjugated (FITC) goat antihuman IgG (dilution 1:800, De Beer Medicals bv, Hilvarenbeek, The Netherlands), FITCconjugated goat anti-human IgA (dilution 1:800, De Beer Medicals), FITC-conjugated goat antihuman IgM (dilution 1:800, Kallestad Lab Inc, Chaska, MN, USA), mouse anti-human IgE (dilution 1:50, Central Lab Red Cross Bloodtransfusion Service (CLB), Amsterdam, The Netherlands), rabbit anti-human complement $\left(\mathrm{C}_{3 \mathrm{c}}, \mathrm{C}_{3 \mathrm{~d}}, \mathrm{C}_{4}\right.$; dilution 1:50, CLB), rabbit antihuman $\mathrm{C}_{\mathrm{lq}}$ (dilution 1:50, CLB) and rabbit antihuman fibrinogen (dilution 1:50, CLB). As negative controls normal rabbit serum (Dakopatts, Denmark) and sections of a macula from a 41-year-old subject, which did not exhibit any abnormalities by conventional light microscopy, were used. As positive controls frozen sections of human skin and kidney from subjects with known immune complex diseases were routinely used at regular time intervals. After incubation with the primary antibodies, the slides were washed and if necessary incubated with FITCconjugated horse anti-rabbit secondary antibodies by the indirect method (polyclonals: complement, fibrinogen, $\mathrm{C}_{\mathrm{lq}}, \mathrm{C}_{3}$ ) or with FITCconjugated rabbit anti-mouse antibodies (monoclonal: IgE). After washing, the sections were covered with glycerin phosphate buffer. The sections were examined with a fluorescence microscope (Zeiss, Oberkochen, Germany) with epi-illumination (excitation: BP $450-490 \mathrm{~nm}$; dichroic mirror: CBS $510 \mathrm{~nm}$; emission: $\mathrm{BP}$ 520-560 nm). The immunoreaction was considered to be positive when a bright extracellular granular fluorescence was present in a linear or patchy pattern. Three sections of every macula

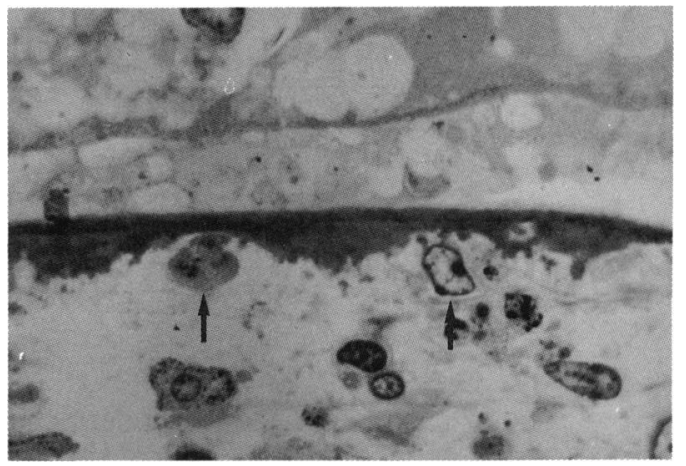

Figure 2 Light microscopy of a $1 \mu \mathrm{m}$ plastic section of a macula with a fibrovascular scar. Macrophages, heavily loaded with phagolysosomes and residual bodies, can be seen on the outer side of Bruch's membrane (arrows). The macrophages can be seen preferentially in places where the capillaries of the choriocapillaris were probably situated before they became atrophic. However, between the intercapillary pillars, Bruch's membrane is thinner than normal for that age. On the right a gap in Bruch's membrane has almost developed. The RPE has degenerated completely and the photoreceptors have disappeared. (Toluidine blue, $\times 350$.)

were stained with haematoxylin and azofloxin for normal light microscopy to detect BLD and drusen.

To reveal the possible presence of immune complexes, seen as dense deposits, and to confirm the presence of macrophages and ruptures in Bruch's membrane, we fixed the opposite half of several maculae with a mixture of $4 \%$ formaldehyde $/ 1 \%$ glutaraldehyde (for 24 hours, $\mathrm{pH}$ 7·4). After fixation the tissue was divided into three smaller parts and embedded in epoxy resin (LX 112, Ladd Research Industries Inc, Burlington, VT, USA) for transmission electron microscopy, as previously described. ${ }^{2}$ Semithin sections ( $1 \mu \mathrm{m}$ thick) were cut with a glass knife and stained with toluidine blue for light microscopy. Ultrathin sections (30-40 $\mathrm{nm}$ thick) were cut with a diamond knife on an ultrotome (LKB, Stockholm, Sweden) and mounted on unfilmed 200-mesh copper grids. After staining with uranyl acetate and lead citrate, the ultrathin sections were examined with a transmission electron microscope (Zeiss TEM 902, Oberkochen, Germany), with an acceleration voltage of $80 \mathrm{kV}$. Micrographs were made on sheet film (Kodak SO 163, Eastman Kodak, Rochester, NY, USA).

\section{Results}

In 16 of the 20 maculae hard drusen and BLD of varying thickness (class 1 to $3^{3}$ ) were present in the haematoxylin and azofloxin stained frozen sections. All sections exhibited thickening of Bruch's membrane (class 2 to 3$).{ }^{3}$ In two maculae geographic atrophy was present and in one macula a disciform scar was seen.

Hard drusen remained unstained with antibodies against $\operatorname{IgG}, \operatorname{IgA}$, IgE, IgM, fibrinogen, $\mathrm{C}_{\mathrm{lq}}$, and $\mathrm{C}_{3 \mathrm{c}}$. In three sections some of the drusen exhibited partial faint granular or homogeneous staining for complement factors. The BLD showed a local faint granular staining for complement factors in three maculae and for fibrinogen in two other maculae. In the negative controls no immunofluorescence was seen in these places.

In one macula there was a combination of 


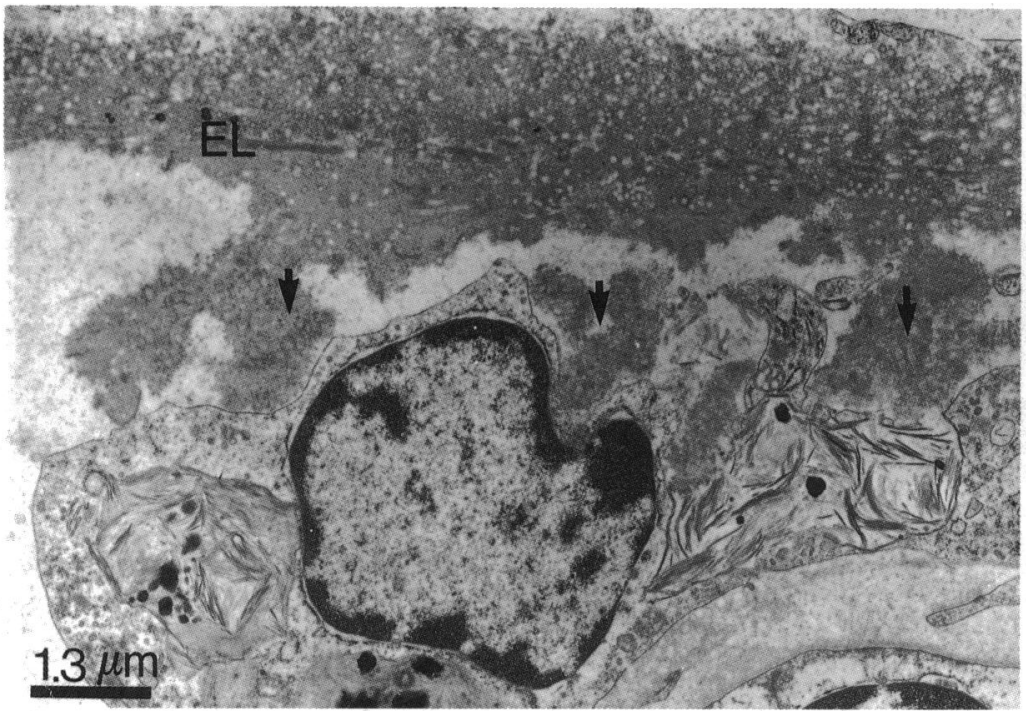

Figure 3 Electron micrograph of a macrophage, filled with phagolysosomes adjacent to Bruch's membrane (top) on the side of the

choriocapillaris. On the left Bruch's membrane has disappeared up to the elastic layer $(E L)$. The material seen between the pseudopodal extensions of the cell is mostly long spacing collagen (arrows), which has come loose from the outer collagenous zone of Bruch's membrane.

Figure 4 Electron micrograph of a macrophage, which envelops material from Bruch's membrane (top) from the side of the choriocapillaris (arrows).

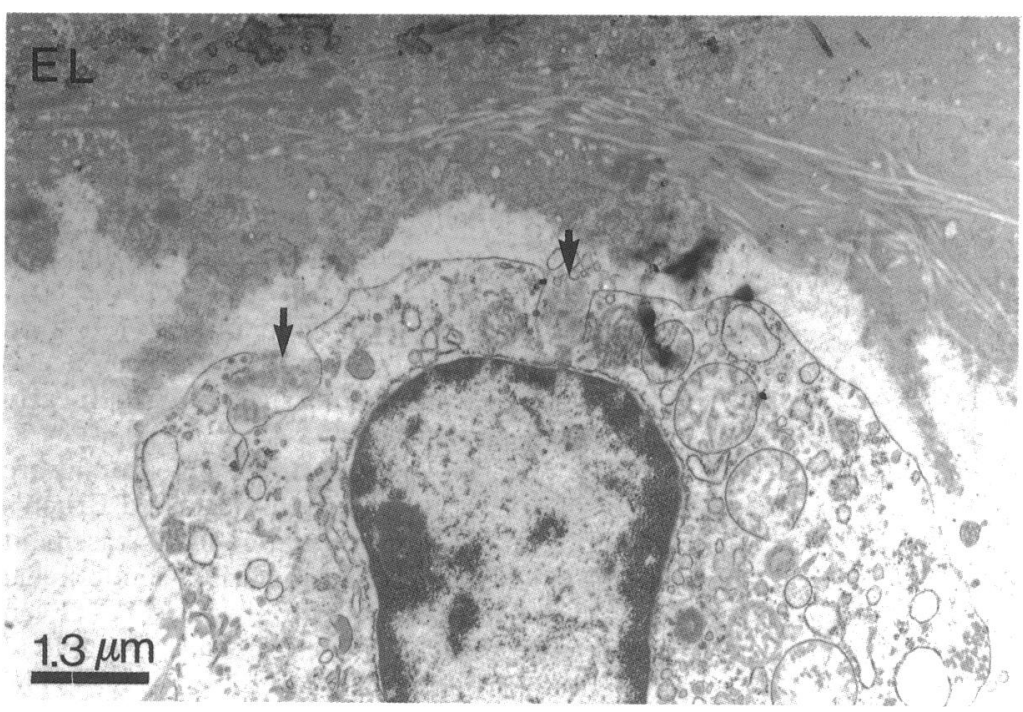

staining of the BLD for complement factors and fibrinogen. Bruch's membrane and the lipofuscin granules of the RPE exhibited autofluorescence, which was also seen in the negative controls.

A linear rim of the OCZ of Bruch's membrane adjacent to the choriocapillaris was positive for fibrinogen (16/20 maculae) (Fig la, complement (12/20 maculae) (Fig 1b), $\mathrm{C}_{\mathrm{lq}}$ (1/20 macula), $\mathrm{C}_{3 \mathrm{c}}$ (1/20 macula) and IgM (1/20 macula). The latter three positive reactions were in different maculae. In the macula with a disciform scar light microscopic observations of semithin sections revealed several gaps and abnormally thin areas in Bruch's membrane (Fig 2).

On electron microscopic observation, cells phages were seen in the vicinity of these places. These cells contained phagolysosomes with digestion products. The images are suggestive of phagocytosis of the OCZ of Bruch's membrane by these cells (Figs 3 and 4). Bruch's membrane was destroyed from the side of the choriocapillaris on, up to the elastic layer. Calcifications in the elastic layer seemed to have stopped the seen (Fig 5).

Between the infoldings of the plasma memwith the morphological characteristics of macrophagocytic cells, but several complete gaps were

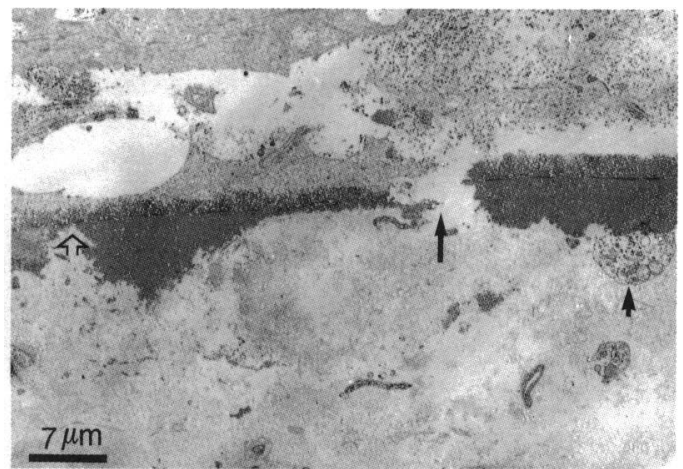

Figure 5 Electron micrograph of a severely thinned Bruch's membrane in a macula with a disciform scar. Notice the thin area in Bruch's membrane on the left (open arrow), the total gap in the middle (large arrow), which is probably a

postmortem artefact due to the thinning up to the elastic layer and a macrophage on the right (small arrow). The RPE is totally degenerated.

branes of the phagocytic cells, material which resembled that of the OCZ could be seen; it consisted mainly of long spacing collagen (Figs 3 , 4, and 6). Unfortunately this macula with macrophages was aldehyde fixed. Therefore immunoreactions could not be investigated adequately. Thus the negative results of immunofluorescence found for this single macula were unreliable.

In another macula, electron microscopy revealed a clear example of diapedesis of an endothelial cell of the choriocapillaris. A cytoplasmic sprout of the cell pointed in the direction of Bruch's membrane (see Fig 8).

\section{Discussion}

Circulating immune complexes are in general associated with many systemic immune diseases, such as certain types of arthritis, glomerulonephritis, or systemic vasculitis syndromes. ${ }^{11}$ Patients with ARMD usually do not exhibit systemic manifestations of immune complex diseases. ${ }^{1}$

Humoral immunity against BLD, drusen, or deposits of cellular debris in Bruch's membrane with circulating immune complexes and elevated levels of serum IgG, IgA, IgM, IgE, or complement has, to the best of our knowledge, not been investigated in eyes with early or advanced stages of ARMD.

Immune complex deposits within the drusen or BLD could not be demonstrated unequivo-

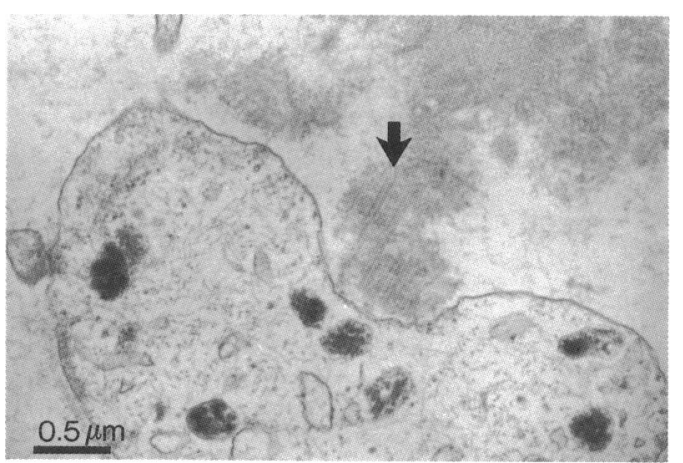

Figure 6 Electron micrograph of a macrophage adjacent to Bruch's membrane (top) on the side of the choriocapillaris. The phagocytised material consists mainly of long spacing collagen (arrow). 


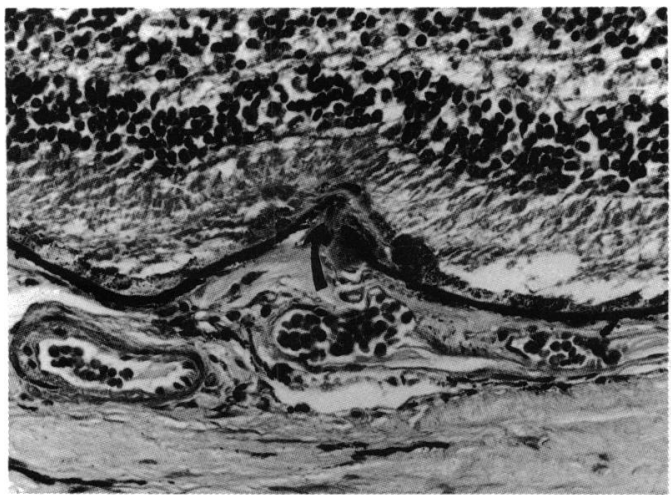

Figure 7 Light microscopic image of a paraffin section of a macula stained for calcium phosphates (Von Kossa). Notice the break in the highly calcified Bruch's membrane, with vascular ingrowth from the choriocapillaris into the sub-RPE space (curved arrow) (magnification $\times 220$ ).

cally because of the faint and only local staining. Almost all sections, including those from younger subjects, revealed linear deposits of complement and fibrinogen in the OCZ of Bruch's membrane, adjacent to the choriocapillaris, suggesting deposition derived from the choriocapillaris, possibly as a result of leakage through the fenestrated endothelium. The presence of complement and fibrinogen without immunoglobulins is not considered to be a sign of immune complex disease.

Human vascular endothelial cells synthesise and secrete complement factors. Both activators $\left(\mathrm{C}_{3}\right)$ and inhibitors (factor $\mathrm{H}$ ) are produced and regulated by cytokines (interleukin $1, \gamma$ interferon. ${ }^{1011}$ Therefore the observed deposits of $\mathrm{C}_{3}$ probably originated from the circulation. This explains the linear deposition of complement along the choriocapillaris. The fibrinogen was probably derived from leakage of the capillaries.

Lysosomal destruction of complement and immunoglobulins has been described. Lysosomal enzymes, such as elastase and collagenase from neutrophilic granulocytes and maybe also from macrophages ${ }^{8}$ may cause tissue destruction as well as removal of the immune complexes. ${ }^{12}$

Figure 8 Electron micrograph of an endothelia cell of the choriocapillaris (bottom), which has penetrated its basement $(B)$ and projects a cytoplasmic sprout towards the OCZ of Bruch's membrane (top).

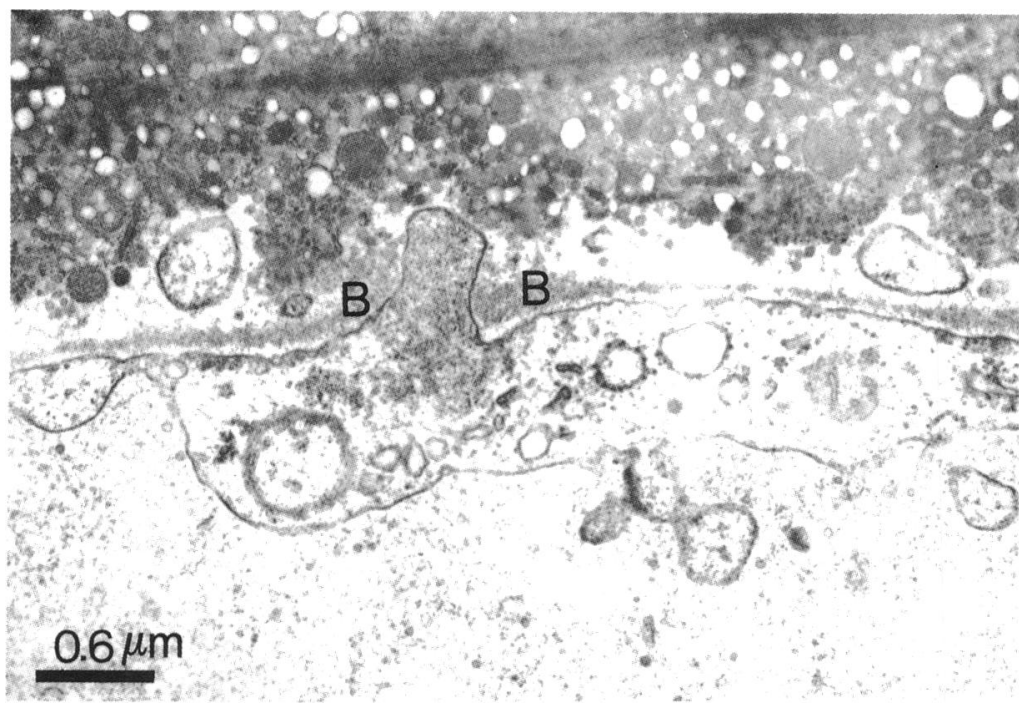

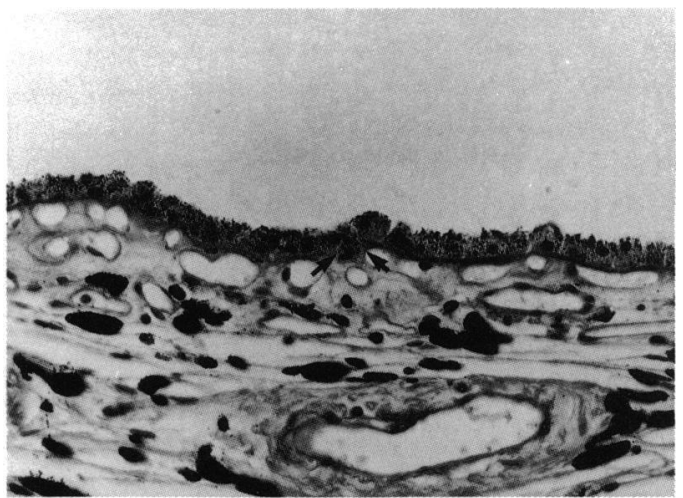

Figure 9 Light microscopic image of a paraffin section of a human macula. Notice the RPE cell, which seems to have penetrated Bruch's membrane and is now located hal fway to the choriocapillaris (arrows). (Mallory stain, magnification $\times 220$.)

observed, as was found in our study. However, to remove all of this material more macrophages or neutrophilic granulocytes should be present in this tissue. Moreover, in the macula of the 41year-old subject similar deposits of complement and fibrinogen were found. This is an argument against the theory of immune complexes as a causal factor in the development of ARMD.

Our electron micrographs show phagocytic cells close to perforations in Bruch's membrane in a macula with a fibrovascular scar, as has previously been described by others. ${ }^{8}$ These cells exhibited the morphological characteristics of macrophages, with numerous phagolysosomes and residual bodies. ${ }^{8}$ The electron micrographs clearly suggest that these cells participate in the digestion of Bruch's membrane from the side of the OCZ. Thus, a pathway for subretinal neovascularisation, which is the initial phase in the formation of a fibrovascular scar, is formed. There appeared to be a preference for phagocytosis of the long spacing collagen, which is often seen in the outer collagenous zone of Bruch's membrane. ${ }^{214}$ However, the presence of this material in a high percentage of the maculae ${ }^{2}$ compared with the low prevalence of disciform macular degeneration ${ }^{3}$ makes it questionable whether this is the only causal factor involved in the formation of gaps in Bruch's membrane. Proteins changed because of interaction with free radicals, which are formed under the influence of ultraviolet radiation, cannot be recognised as autologous material. This might be another explanation for this phagocytic action.

Other factors for macrophage attraction, such as immune complex deposits or foreign substances in Bruch's membrane, such as the deposition of phospholipids and neutral fats containing PVC plasticisers with advancing age, could also be involved. ${ }^{15} 16$ With electron microscopy, immune complexes can be identified ${ }^{12}$ but were not seen in this study. Moreover, the presence of macrophages is not a consistent finding in early and advanced ARMD.

Other causes of gaps in Bruch's membrane are mechanical breaks in a highly calcified Bruch's membrane (Fig 7) or perforation of the basement membrane of the choriocapillaris by endothelial cells (Fig 8), as described previously. ${ }^{817-19}$ Even single RPE cells have been shown to be able to penetrate Bruch's membrane (Fig 9). ${ }^{1020}$ The 
initial cause of this endothelial outgrowth could be the absence of vascular inhibiting factors, which are normally produced by the RPE cells, ${ }^{21} 22$ the presence of angiogenic stimulating factors from macrophages, ${ }^{11823}$ or a reaction to ischaemia in the outer retina or choroid.

From our study we can conclude that definite immune complex deposits cannot be found in maculae with BLD or drusen. Linear deposition of fibrinogen and complement was found on the choriocapillaris side of the outer collagenous zone of Bruch's membrane. These deposits are considered to be non-specific and not typical for immune complex diseases. Accumulation of unknown or changed proteins in the outer collagenous zone of Bruch's membrane might be a cause of activation of macrophages, as seen in the vicinity of perforations in Bruch's membrane. However, the presence of macrophages was not a consistent finding in early and late stages of ARMD and thus they are not likely to be the sole factor in the development of ARMD.

We thank N Stouten, C J Vissers, and A Timmermans for their technical assistance.

1 Leibowitz HM,Krueger DE, Maunder LR, Milton RC, Kini MM, Kahn HA, et al. The Framingham Eye Study Monograph; an ophthalmological and epidemiological study of cataract, glaucoma, diabetic retinopathy, macular degeneracataract, glaucoma, diabetic retinopathy, macular degeneration, and visual acuity in a general population of 2631

2 Van der Schaft TL, de Bruijn WC, Mooy CM, Ketelaars DAM, de Jong PTVM. Is basal laminar deposit unique for age-related macular degeneration? Arch Ophthalmol 1991; 109: 420-5.

3 Van der Schaft TL,Mooy CM, de Bruijn WC, Oron FG, Mulder PGH, de Jong PTVM. Histologic features of the early stages of age-related macular degeneration. Ophthalmology 1992; 99: 278-86.

4 Sarks SH. Aging and degeneration in the macular region: a clinico-pathological study. $\mathrm{Br} \mathcal{F}$ Ophthalmol 1976; 60: clinico-pat.

5 Green WR, McDonnel PJ, Yeo JH. Pathologic features of senile macular degeneration. Ophthalmology 1985; 92: 615-27.

6 Coffey AJH, Brownstein S. The prevalence of macular drusen in postmortem eves. Am $\mathcal{F}$ Ophthalmol 1986; 102: 164-71.

Löffler KU, Lee WR. Basal linear deposit in the human macula. Graefes Arch Clin Exp Ophthalmol 1986; 224: 493-501.

8 Killingsworth MC, Sarks JP, Sarks SH. Macrophages related to Bruch's membrane in age-related macular degeneration. to Bruch's membrane in age-related macular degeneration.
Eye 1990; 4: 613-21.

9 Nishimura T, Goodnight R, Prendergast RA, Ryan SJ. Activated macrophages in experimental subretinal neovascularization. Ophthalmologica 1990; 200: 39-44.

10 Penfold P, Killingsworth M, Sarks SH. An ultrastructural study of the role of leucocytes and fibroblasts in the breakdown of Bruch's membrane. Aust f Ophthalmol 1984; 12: 23-31.

11 Faure JP, Bloch-Michel E, Le Hoang P, Vadot E. Immunopathologie de l'oeil. Paris: Societe Francaise d'Ophthalmologie et Masson, 1988: 291-327.

12 Wolff $\mathrm{HH}$, Maciejewski W, Scherer R, Braun-Falco C. Immunoelectronmicroscopic examination of early lesions in histamine induced immune complex vasculitis in man. Brf Dermatol 1978; 99: 13-22.

13 Pollack A, Korte GE, Weitzner AL, Henkind P. Ultrastructure of Bruch's membrane after krypton laser photocoagulature of Bruch's membrane after krypton laser photocoagula-
tion. II. Repair of Bruch's membrane and the role of tion. II. Repair of Bruch's membrane and the

macrophages. Arch Ophthalmol 1986; 104: 1377-82.
14 Killingsworth MC. Age-related components of Bruch's membrane in the human eye. Graefes Arch Clin Exp Ophthalmol 987; 225: 406-12.

15 Bird AC. Pathogenesis of retinal pigment epithelial detachment in the elderly; the relevance of Bruch's membrane change. Eye 1991; 5: 1-12.

16 Pauleikhoff D, Harper CA, Marshall J, Bird AC. Aging changes in Bruch's membrane. Ophthalmology 1990; 97 : 171-8.

17 Penfold PL, Provis JM, Bilson FA. Age-related macular degeneration: ultrastructural studies of the relationship of leucocytes to angiogenesis. Graefes Arch Clin Exp Ophthalmol 1987; 225: 70-6.

18 Pollack A, Korte GE, Weitzner AL, Henkind P. Ultrastructure of Bruch's membrane after krypton laser photocoagulation. I: Breakdown of Bruch's membrane. Arch Ophthalmol tion. I: Breakdown

19 Heriot WJ, Henkind P, Bellhorn RW, Burns MS. Choroidal neovascularization can digest Bruch's membrane. A prior break is not essential. Ophthalmology 1984; 91: 1603-8.

20 Grindle CFJ, Marshall J. Ageing changes in Bruch's membrane and their functional implications. Trans Ophthalmol Soc UK 1978; 98: 172-5.

21 Glaser BM, Campochiaro PA, Davis JL, Sato Misao. Retinal pigment epithelial cells release inhibitor of neovascularization. Arch Ophthalmol 1985; 103: 1870-5.

22 Campochiaro PA, Glaser BM. Endothelial cells release a chemo attractant for retinal pigment epithelial cells in vitro. Arch Ophthalmol 1985; 103: 1876-80.

23 Penfold PL, Provis JM, Madigan MC, van Driel D, Billson FH. Angiogenesis in normal human retinal development: the involvement of astrocytes and macrophages. Graefes Arch Clin Exp Ophthalmol 1990; 228: 255-63. 